\title{
In breast cancer, a high ratio of tumour-infiltrating intraepithelial CD8+ to FoxP3+ cells is characteristic for the medullary subtype
}

\author{
David Anz, ${ }^{1,2}$ Stephan Eiber, ${ }^{1}$ Christoph Scholz, ${ }^{3}$ Stefan Endres, ${ }^{1}$ Thomas Kirchner, ${ }^{4}$ \\ Carole Bourquin ${ }^{1,5}$ \& Doris Mayr ${ }^{4}$ \\ ${ }^{1}$ Centre of Integrated Protein Science Munich (CIPS-M), Division of Clinical Pharmacology, ${ }^{2}$ Medizinische Klinik \\ Innenstadt, ${ }^{3}$ Department of Obstetrics and Gynaecology, Ludwig Maximilian University, ${ }^{4}$ Department of Pathology, Ludwig \\ Maximilian University, Munich, Germany, and ${ }^{5}$ Department of Pharmacology, University of Fribourg, Switzerland
}

\begin{abstract}
Aims: Medullary breast cancer (MBC) is a biologically distinct subtype of breast cancer characterized by prominent lymphocytic infiltrates and a favourable clinical outcome. Tumour-infiltrating CD $8+$ effector $\mathrm{T}$ cells may contribute to the good prognosis of this type of cancer; however, certain subtypes of lymphocyte, such as FoxP3+ regulatory $\mathrm{T}$ cells (Tregs), can also suppress antitumour immunity.

Methods and results: We determined tumour infiltration by FoxP3+, CCL22+ and CD8+ cells in paraffinembedded sections of $\mathrm{MBC}$, and, as a reference, in samples of grade 3 ductal, lobular and mucinous breast cancer. All analysed MBCs were strongly infiltrated by FoxP3+ cells, whereas only weak infiltrates were detected in ductal or lobular breast cancer. This finding
\end{abstract}

was unexpected, given the good prognosis of MBC. Strikingly, the number of CD8+ T cells exceeded the number of FoxP3+ cells in $\mathrm{MBC}$ (ratio of $\mathrm{CD} 8+$ to FoxP3+ cells of 2.6), whereas equal amounts of both cell types were found in ductal breast cancer (ratio of CD8+ to FoxP3+ cells of 1.1). In both types of breast cancer, we also detected cells expressing the Tregattracting chemokine CCL22.

Conclusions: In breast cancer, a predominance of tumour-infiltrating CD8+ over FoxP3+ cells was observed in MBC. Thus, the ratio of $\mathrm{CD} 8+$ to FoxP3+ cells rather than the absolute number of intratumoral FoxP $3+$ cells may be predictive for the clinical outcome of cancer.

Keywords: breast cancer, CCL22, cytotoxic T cells, FoxP3, lymphocytes, medullary breast cancer, regulatory T cells Abbreviations: DBC, ductal breast cancer; ELISA, enzyme-linked immunosorbent assay; MBC, medullary breast cancer; PCR, polymerase chain reaction; Treg, regulatory T cell

\section{Introduction}

Medullary breast cancer (MBC) is a particularly interesting subtype, as it is characterized by less frequent lymph node metastasis ${ }^{1}$ and good clinical outcome. ${ }^{2}$ Histologically, it consists of large sheets of poorly differentiated tumour cells appearing in a syncytial

Address for correspondence: D Mayr, Department of Pathology, Thalkirchner Strasse 36, 80337 Munich, Germany.

e-mail: doris.mayr@med.uni-muenchen.de growth pattern and prominent lymphocytic infiltration of the tumour stroma. ${ }^{3}$ Several authors have suggested that a high number of tumour-infiltrating lymphocytes may help to limit tumour burden and thus contribute to good prognosis in $\mathrm{MBC}^{3-6}$ Tumour-infiltrating lymphocytes, however, represent a heterogeneous group of immune cells composed of both effector cell subsets and immunosuppressive cells.

Regulatory T cells (Tregs) represent a population of immunosuppressive cells that are capable of inhibiting both innate and adaptive immunity. A key function of 
Tregs is the suppression of immune responses directed against self-antigens, and these cells are therefore crucial for the prevention of autoimmunity. ${ }^{7}$ However, Tregs also inhibit the cytotoxic activity of CD8+ T cells against malignant tumours, and many studies have shown that Tregs play a crucial role in tumour-induced immunosuppression. ${ }^{8}$ The transcription factor FoxP3 is, so far, the most specific marker for the identification of human Tregs, although it can also be expressed by other lymphocytes upon activation. ${ }^{9}$ Infiltration of tumours by FoxP3+ Tregs has been observed in different types of human cancer, and is associated with poor prognosis. ${ }^{10,11}$ The recruitment of Tregs to the tumour tissue depends on specific mediators that attract these cells to the tumour site. The chemokine CCL22 represents one important factor ${ }^{12}$ that can mediate migration of FoxP3+ Tregs to the tumour site. ${ }^{10}$

Although a high number of intratumoral lymphocytes is consistently seen in MBC, infiltration by FoxP3+ Tregs in this type of breast cancer has not been investigated. In this study, we analysed paraffin-embedded material of 13 MBCs, 34 ductal breast cancers (DBCs) and seven lobular or mucinous breast cancers for the presence of FoxP3+ and CD8+ lymphocytes and for CCL22+ cells in the tumour tissue. Interestingly, all analysed MBCs were strongly infiltrated by both FoxP3+ and CD8+ cells. CCL22 was detected in all tumours, and was mainly expressed by tumour-infiltrating immune cells and, in a few cases, by the cancer cells themselves. We further determined the ratio of effector $\mathrm{T}$ cells to Tregs in the tumours, and found that the number of intratumoral CD8+ cells clearly exceeded the number of FoxP3+ cells in MBCs but not in DBCs. The high ratio of $\mathrm{CD} 8+$ to FoxP3+ cells may thus contribute to the relatively good prognosis of patients with MBC.

\section{Materials and methods}

\section{PATIENTS}

Paraffin-embedded material of 54 breast cancer patients, including 13 MBCs, 34 DBCs, four lobular breast cancers and three mucinous carcinomas, was used for histological analysis (Table 1). All cases were retrieved from the archives of the Institute of Pathology of the Ludwig Maximilian University, Munich, Germany. The age of the patients ranged from 26 to 80 years. All patients were treated surgically between 2004 and 2008 at the same institution (Department of Obstetrics and Gynaecology Maistrasse, University of Munich). Tumour typing and staging were performed according to the classification of the International Union against Cancer. $\mathrm{MBC}$ was diagnosed when the following five features were present: (i) well-circumscribed tumour borders; (ii) lymphocytic reaction diffusely in the tumour and in the periphery; (iii) syncytial pattern of tumour cell growth; (iv) high nuclear grade; and (v) high mitotic activity. The DBC tissues used in this study were all grade 3. Tissue sections were reviewed by independent investigators at the Institute of Pathology of the Ludwig Maximilian University, Munich.

\section{IMMUNOHISTOLOGY}

All paraffin-embedded specimens were cut at 2-3 $\mu \mathrm{m}$ and mounted on SuperFrost Plus microscope slides (Menzel Gläser, Braunschweig, Germany). After deparaffinization and rehydration, immunohistochemical assays were performed with standard methods. The following antibodies were used: mouse anti-human FoxP3 (1:100; Abcam, Cambridge, UK), rabbit antihuman CCL22 (1:350; Peprotech, Rocky Hill, NJ, USA), and rabbit anti-human CD8 (1:50; Thermo Fisher Scientific, Fremont, CA, USA). Appropriate horseradish peroxidase-conjugated and alkaline phosphatase-conjugated secondary antibodies were used for detection. The amounts of tumour-infiltrating FoxP3+, CCL22+ and CD8+ cells were evaluated semiquantitatively by analysing the complete surface of the tissue section. The following scoring system was used: -, no infiltrating cells; +, low numbers of infiltrating cells; ++ , medium numbers of infiltrating cells; and +++ , high numbers of infiltrating cells. For analysis of the ratio of $\mathrm{CD} 8+$ to FoxP3+ cells, absolute cell numbers infiltrating the tumour epithelium were counted in digital images derived from CD8-stained and FoxP3stained serial sections, and the ratio was calculated for each pair. Digital images were obtained at 20-fold or 40-fold magnification (Axiovert Inverse Microscope; Carl Zeiss, Jena, Germany), and processed with ADoBE Рнотоsнор for adjustment of contrast and size.

CELL CULTURE AND ENZYME-LINKED

IMMUNOSORBENT ASSAY (ELISA)

The human breast cancer cell lines MCF7 and MDAMB-231 were maintained in Dulbecco's modified Eagle's medium supplemented with 10\% fetal bovine serum, $1 \%$ L-glutamine, $1 \mathrm{U} / \mathrm{ml}$ penicillin, and $0.1 \mathrm{mg} / \mathrm{ml}$ streptomycin (all PAA Laboratories, Coelbe, Germany). Dendritic cells were generated by using peripheral blood mononuclear cells from peripheral blood of healthy donors and by culturing the adherent fraction in the presence of granulocyte-macrophage colony-stimulating factor $(1000 \mathrm{U} / \mathrm{ml})$ and interleukin-4 (500 U/ml) for 24 h. Supernatants were assayed 
Table 1. Patient and tumour characteristics

\begin{tabular}{|c|c|c|c|c|}
\hline & \multicolumn{4}{|c|}{ Histological subtype } \\
\hline & Medullary & Invasive ductal & Lobular & Mucinous \\
\hline Number of samples & 13 & 34 & 4 & 3 \\
\hline Age in years (range) & $49.7(32-71)$ & $54.4(26-80)$ & $53.0(41-73)$ & $65.3(58-78)$ \\
\hline \multicolumn{5}{|l|}{ TNM stage } \\
\hline $\mathrm{T} 1$ & 3 & 9 & 1 & 1 \\
\hline $\mathrm{T} 2$ & 9 & 13 & 3 & 1 \\
\hline T3 & 1 & 8 & 0 & 1 \\
\hline T4 & 0 & 4 & 0 & 0 \\
\hline NO & 8 & 12 & 1 & 1 \\
\hline N1 & 4 & 12 & 1 & 1 \\
\hline N2 & 0 & 7 & 1 & 0 \\
\hline N3 & 0 & 3 & 1 & 0 \\
\hline $\mathrm{Nx}$ & 1 & 0 & 0 & 1 \\
\hline M1 & 0 & 17 & 0 & 0 \\
\hline$M x$ & 13 & 17 & 4 & 3 \\
\hline HER-2/neu+ & 3 & 21 & 0 & 0 \\
\hline \multicolumn{5}{|l|}{ Tumour grade (SBR) } \\
\hline 2 & 1 & 0 & 4 & 3 \\
\hline 3 & 12 & 34 & 0 & 0 \\
\hline
\end{tabular}

SBR, Scarff-Bloom-Richardson Classification

for CCL22 by ELISA (R\&D Systems, Minneapolis, MN, USA). For immunocytology, single-cell suspensions of formalin-fixed cells were embedded in paraffin and stained as described.

\section{STATISTICAL ANALYSIS}

Data are presented as means in bar diagrams or as box plots, and were analysed with unpaired Student's $t$-test. Statistical analysis was performed with GRAPHPAD PRISM software.

\section{Results}

MBC IS STRONGLY INFILTRATED BY FOXP $3+$ CELLS

To determine Treg infiltration in the tumour tissue of patients with MBC, we stained FoxP3+ cells in paraffin-embedded material of 13 individuals and then performed semiquantitative evaluation of the infiltration density. Interestingly, all analysed MBCs were strongly infiltrated by FoxP3+ cells (Figure 1A). All of these cells showed the typical nuclear staining pattern of the Treg-associated transcription factor FoxP3. In contrast, only a few DBCs were infiltrated by FoxP3+ cells, with 30 of 34 analysed tumours containing no or low amounts of cells. Low numbers of FoxP $3+$ cells were also observed in the four lobular breast cancers and the three mucinous carcinomas. In all breast cancer tissues, two different types of infiltration by FoxP3 + cells were observed: first, infiltration into the tumour epithelium with direct contact with cancer cells (Figure 1B); and second, the presence of FoxP3+ cells in the tumour stroma or in lymphoid clusters that were located either in or around the tumour tissue. Both patterns were observed in MBCs and DBCs, but 

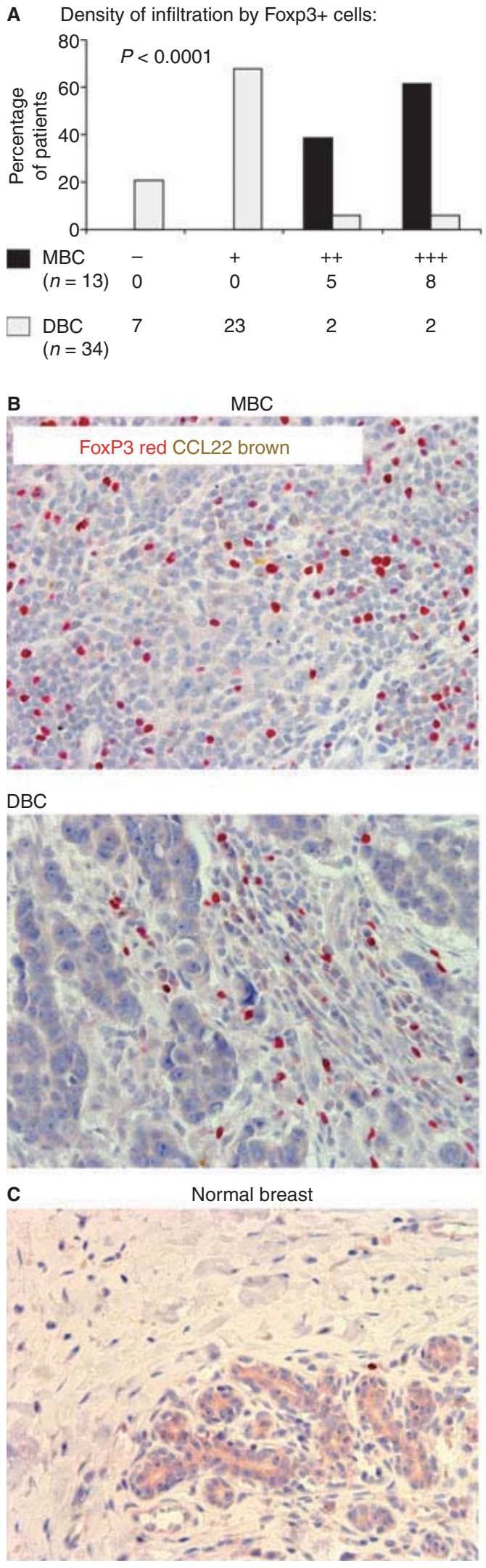

lymphoid clusters were more frequent in MBCs. In contrast to the tumour area, FoxP3+ cells were almost absent from the adjacent healthy breast tissue (Figure 1C). In conclusion, MBCs are infiltrated by high numbers of FoxP3+ cells, whereas other subtypes of breast cancer contain few of these immunosuppressive cells.

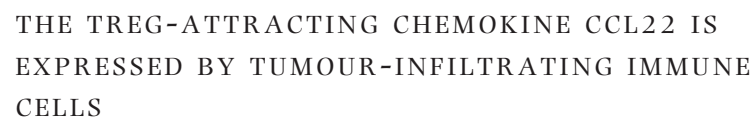

Recruitment of Tregs to malignant tumours can be mediated through the chemokine CCL22. ${ }^{10}$ To determine whether CCL22 may play a role in the migration of Tregs to MBC, we analysed the expression of this chemokine in the respective tumour tissues. CCL22 was mainly expressed by myeloid-shaped immune cells that infiltrated both the tumour stroma and epithelium (Figure 2A). CCL22+ immune cells were detected in all tumours and the adjacent normal breast tissue, and no difference in the frequency of these cells was observed between MBCs and DBCs (Figure 2B). All of the four lobular breast cancer tissues contained numerous CCL22+ cells. In some, but not all, cases, CCL22+ cells were locally associated with accumulations of FoxP3+ cells (Figure 2A). In addition to the expression by immune cells, CCL22 was found to be also expressed by the cancer cells (Figure 3A). Whereas almost none of these cells were found in MBCs or lobular breast cancers, CCL22 expression by cancer cells was detected in $24 \%$ of DBCs (eight of 34). Generally, CCL22+ cancer cells were not found in local association with FoxP3+ Tregs. In some tumours, CCL22 was also strongly expressed by those cancer cells that infiltrated lymphatic vessels (lymphoangiosis carcinomatosis; Figure 3A). In contrast, no CCL22 secretion was detected in vitro from breast cancer cell lines (Figure 3B). In

\footnotetext{
Figure 1. Breast cancer infiltration by FoxP3+ cells. Tumourinfiltrating FoxP3+ lymphocytes were quantified in paraffin-embedded samples of human breast cancer that were double-stained with anti-human FoxP3 (red) and anti-human CCL22 (brown). A, Infiltration by FoxP $3+$ cells was assessed semiquantitatively in medullary breast cancer (MBC, black bars, $n=13$ ) and ductal breast cancer (DBC, white bars, $n=34$ ) specimens (-, no infiltration; +, low infiltration; ++, high infiltration; +++, very high infiltration). Bars indicate the percentage, and the numbers below indicate the absolute number of tumour specimens. B,C, Representative examples of infiltration by FoxP3+ cells are shown for (B) the tumour epithelium of $\mathrm{MBC}$ and $\mathrm{DBC}$, and for $(\mathrm{C})$ the adjacent healthy breast tissue $(\times 40$ magnification).
} 


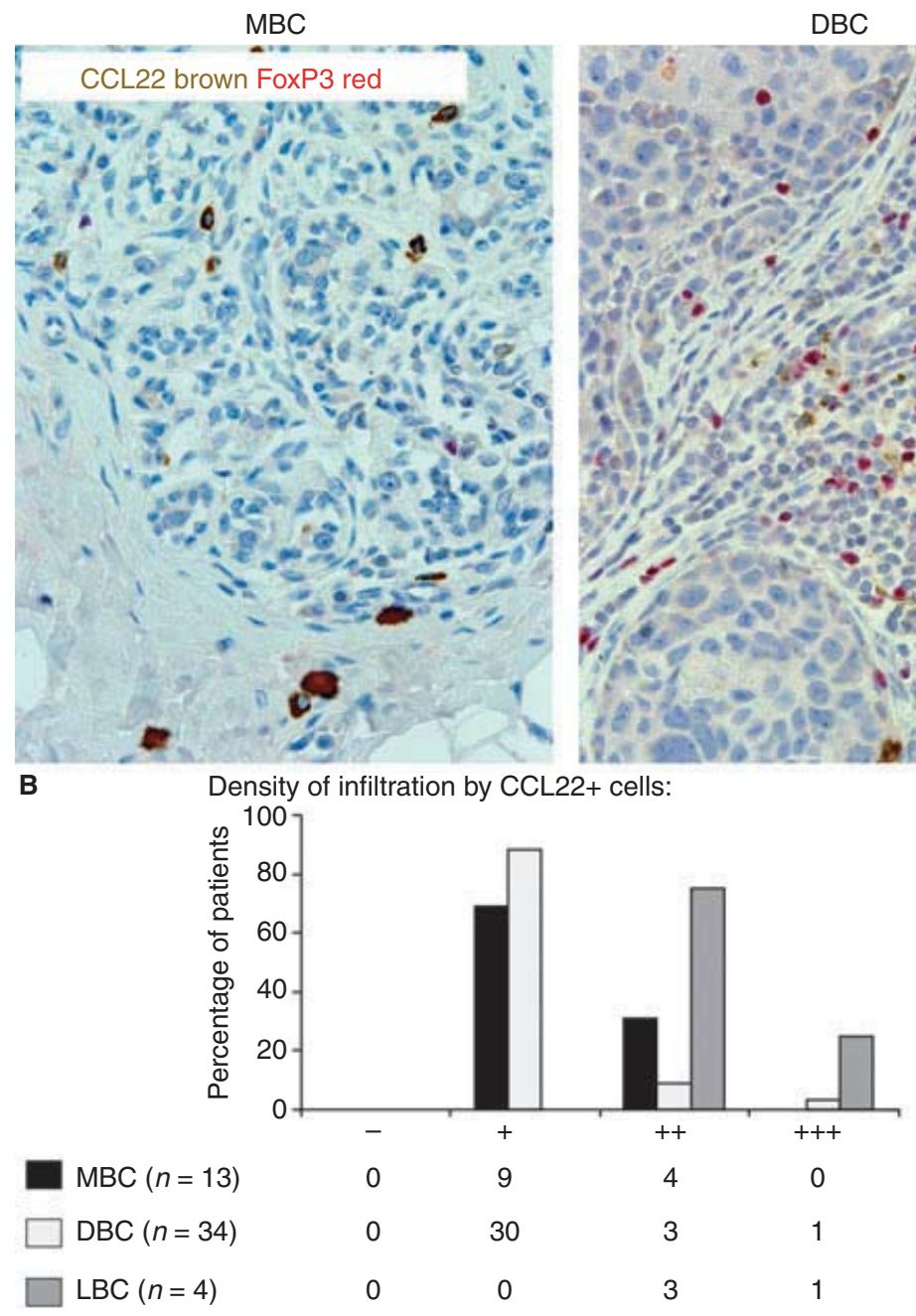

Figure 2. Breast cancer infiltration by CCL22+ immune cells. A, Paraffin-embedded samples of human breast cancer were stained as in Figure 1, and representative examples for CCL22+ immune cells (brown) are shown for medullary breast cancer (MBC), ductal breast cancer (DBC), and adjacent normal breast tissue ( $\times 40$ magnification). B, Infiltration by CCL22+ myeloid-shaped cells was assessed semiquantitatively for $\operatorname{MBC}(n=13$, black bars), DBC ( $n=34$, white bars), and lobular breast cancer (LBC, $n=4$, grey bars) (quantification score as in Figure 1).

summary, CCL22+ immune cells were present in all analysed breast cancer specimens, whereas expression of this chemokine by cancer cells was less frequent and limited to DBCs.

THE NUMBER OF CD $8+$ T CELLS EXCEEDS THE NUMBER OF TUMOUR-INFILTRATING FOXP3 + CELLS IN MBC

The high levels of tumour-infiltrating FoxP3+ cells in MBCs contrast with the relatively good prognosis of this cancer. It is generally accepted that tumour-infiltrating effector T cells, in contrast to Tregs, are associated with a favourable outcome. ${ }^{13,14}$ We therefore analysed the $\mathrm{MBC}$ tissues for the presence of CD8+ effector T cells, and correlated these numbers with the infiltration by FoxP3+ lymphocytes. We detected prominent infiltration of CD8+ T cells in all MBCs (Figure 4A), which is in agreement with previous reports. ${ }^{6,15}$ In contrast, most DBCs and all lobular breast cancers or mucinous carcinomas were infiltrated only weakly by CD8+ cells. In all tumours, CD8+ T cells were located not only in the tumour epithelium but also in the stroma and in lymphoid clusters. To determine the relationship of CD8+ to FoxP3+ cells, we analysed serial sections of either CD8-stained or FoxP3-stained MBC tissues. Interestingly, we found important differences in the ratio of $\mathrm{CD} 8+$ to FoxP3+ cells according to the positions of both cell types in the tumour: in the 
A CCL22+ cancer cells

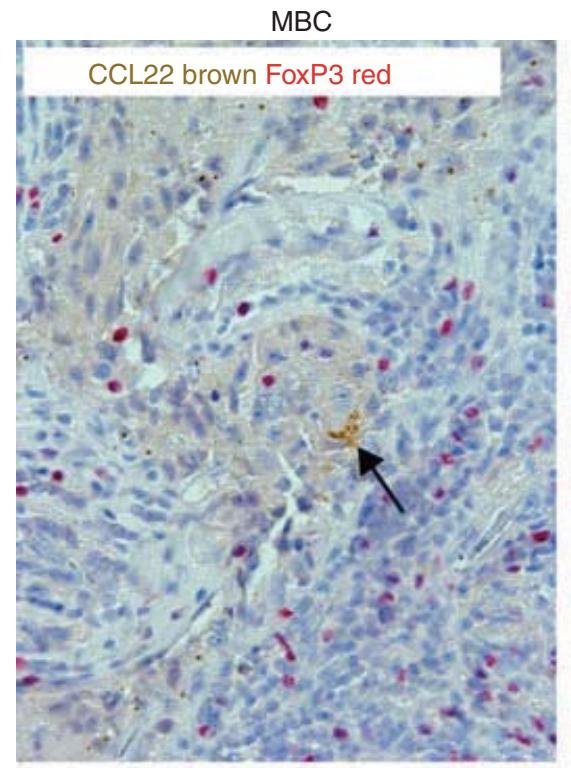

$\mathrm{DBC}$

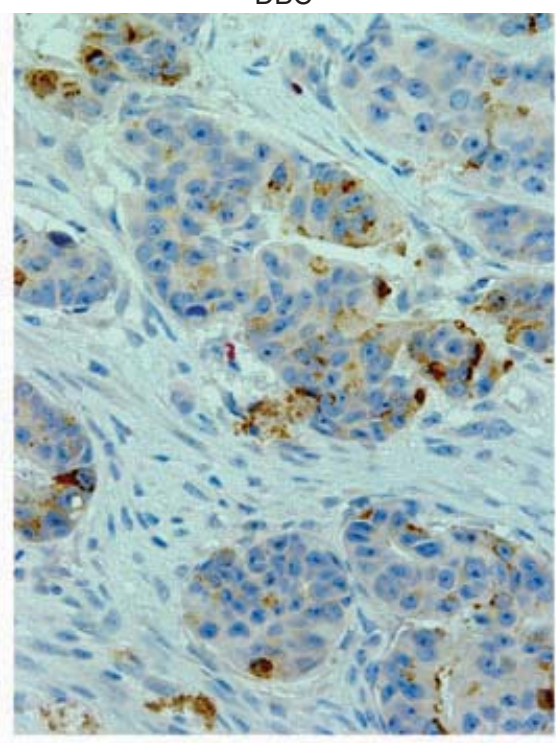

Lymphangiosis c. (DBC)

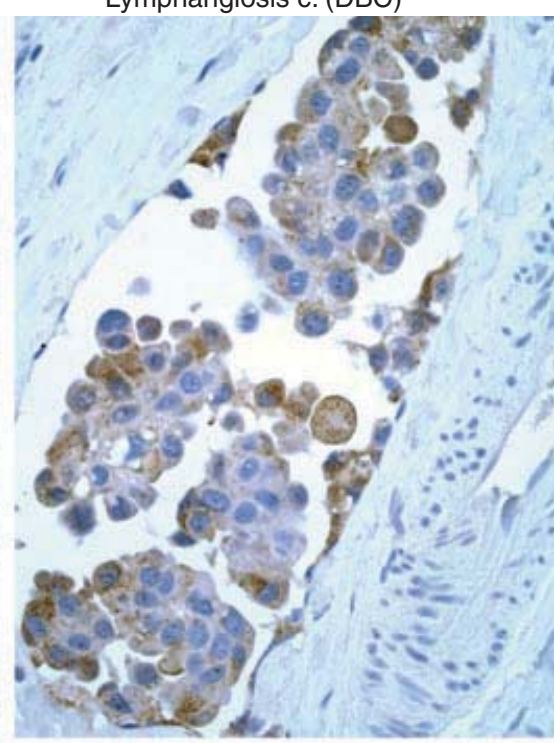

B

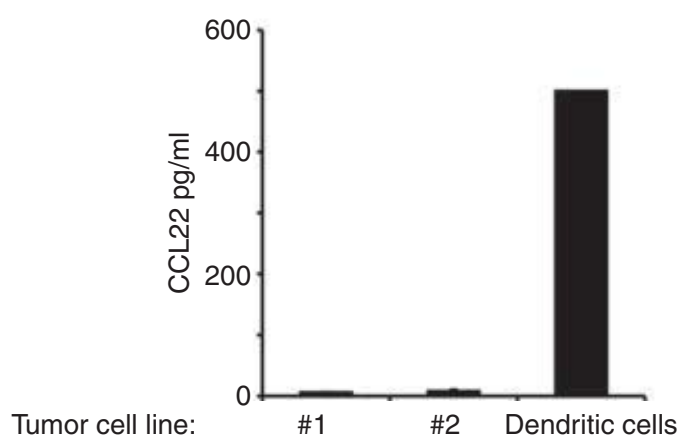

Figure 3. Expression of CCL22 by breast cancer cells. A, Breast cancer samples were prepared as described in Figure 1. Expression of CCL22 by tumour cells (brown) is shown for examples of medullary breast cancer (MBC), ductal breast cancer (DBC) and lymphangiosis carcinomatosis (lymphangiosis c.) within DBC. B, CCL22 was measured in the supernatants of two breast cancer cell lines by enzyme-linked immunosorbent assay, and the supernatants of human dendritic cells were used as a positive control.

tumour epithelium of MBCs, we observed a striking predominance of $\mathrm{CD} 8+\mathrm{T}$ cells, whereas a variable ratio of CD8+ to FoxP3 + cells was seen in lymphoid clusters and the tumour stroma (Figure 4B). To quantify the ratio of $\mathrm{CD} 8+$ to FoxP $3+$ cells in the tumour epithelium of MBCs and DBCs, we counted the numbers of both cell types in congruent visual fields, using serial sections, and calculated the mean of all ratios for both tumour subtypes (Figure 4C). Interestingly, a high ratio of $\mathrm{CD} 8+$ to FoxP3 + cells was confirmed in the tumour epithelium of MBCs (mean ratio of $\mathrm{CD} 8+$ to FoxP3 + cells of 2.6), whereas a significantly lower ratio was found in DBCs (mean ratio of $\mathrm{CD} 8+$ to FoxP3+ cells of 1.1). Thus, in contrast to DBCs, the number of intratumoral $\mathrm{CD} 8+\mathrm{T}$ cells in MBC clearly exceeds the number of FoxP3+ cells.

\section{Discussion}

MBCs consistently feature prominent lymphocytic infiltrates that are associated with good clinical outcome. ${ }^{3}$ In general, grade 3 breast cancer with prominent lymphocytic infiltration has been reported to have a more favourable prognosis. ${ }^{16}$ We show here, for the first time, that MBCs are strongly infiltrated by FoxP3 + cells, a population of lymphocytes with an immunosuppressive function. ${ }^{7}$ High numbers of FoxP3+ cells were observed in all analysed MBC tissues, and both the tumour epithelium and the surrounding stroma were infiltrated by these cells. Although tumour infiltration by FoxP3+ cells has so far not been investigated for MBCs, intratumoral Tregs in DBC or lobular breast cancer have previously been 
A Density of infiltration by CD8+ cells:

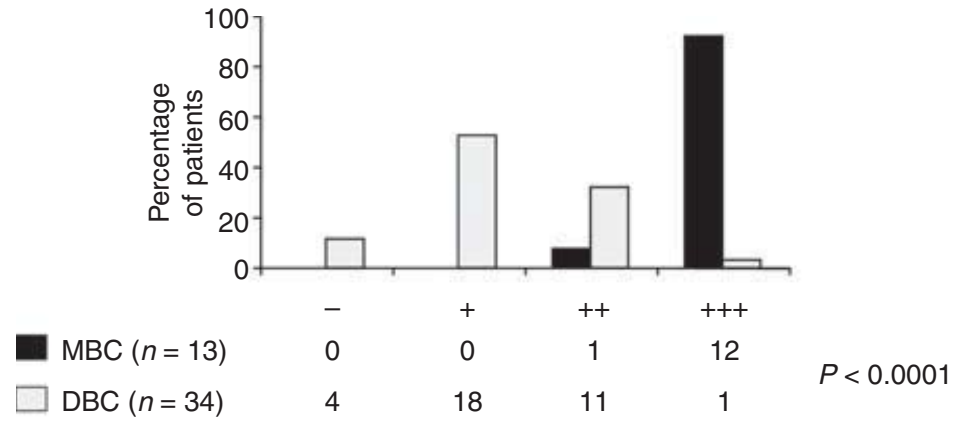

Figure 4. Relationship of tumour-infiltrating $\mathrm{CD} 8+$ to FoxP3+ cells in breast cancer. Serial sections of paraffinembedded breast cancer were stained with either anti-human CD8 (brown) or with antihuman FoxP3 (red) together with CCL22 (brown). A,

Infiltration by $\mathrm{CD} 8+$ cells was assessed semiquantitatively for medullary breast cancer (MBC) ( $n=13$, black bars $)$ and ductal breast cancer (DBC) $(n=34$, white bars) specimens (quantification score as in Figure 1). Bars indicate the percentage, and the numbers below indicate the absolute number of tumour specimens. B, Congruent microscopic fields demonstrate the specific distribution of $\mathrm{CD} 8+$ and FoxP3+ cells in lymphoid clusters (LC) and the tumour epithelium (E) in tissue sections of MBC. C, Intraepithelial CD8+ and FoxP3+ cells in $\mathrm{MBC}$ and DBC were quantified in congruent images of serial sections. The mean ratios of $\mathrm{CD} 8+$ to FoxP $3+$ cells and the ranges for each type of tumour are shown in a box plot.

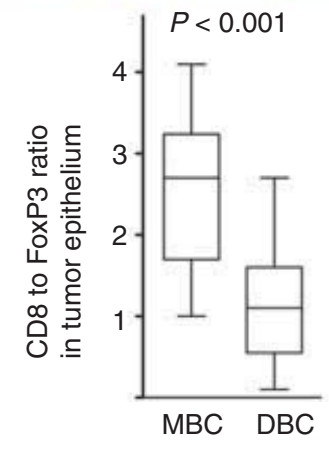

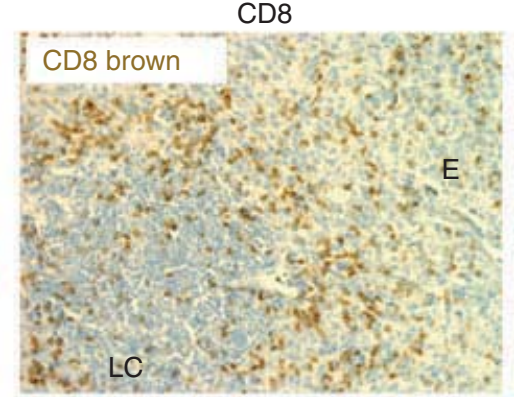

c

\section{FoxP3}
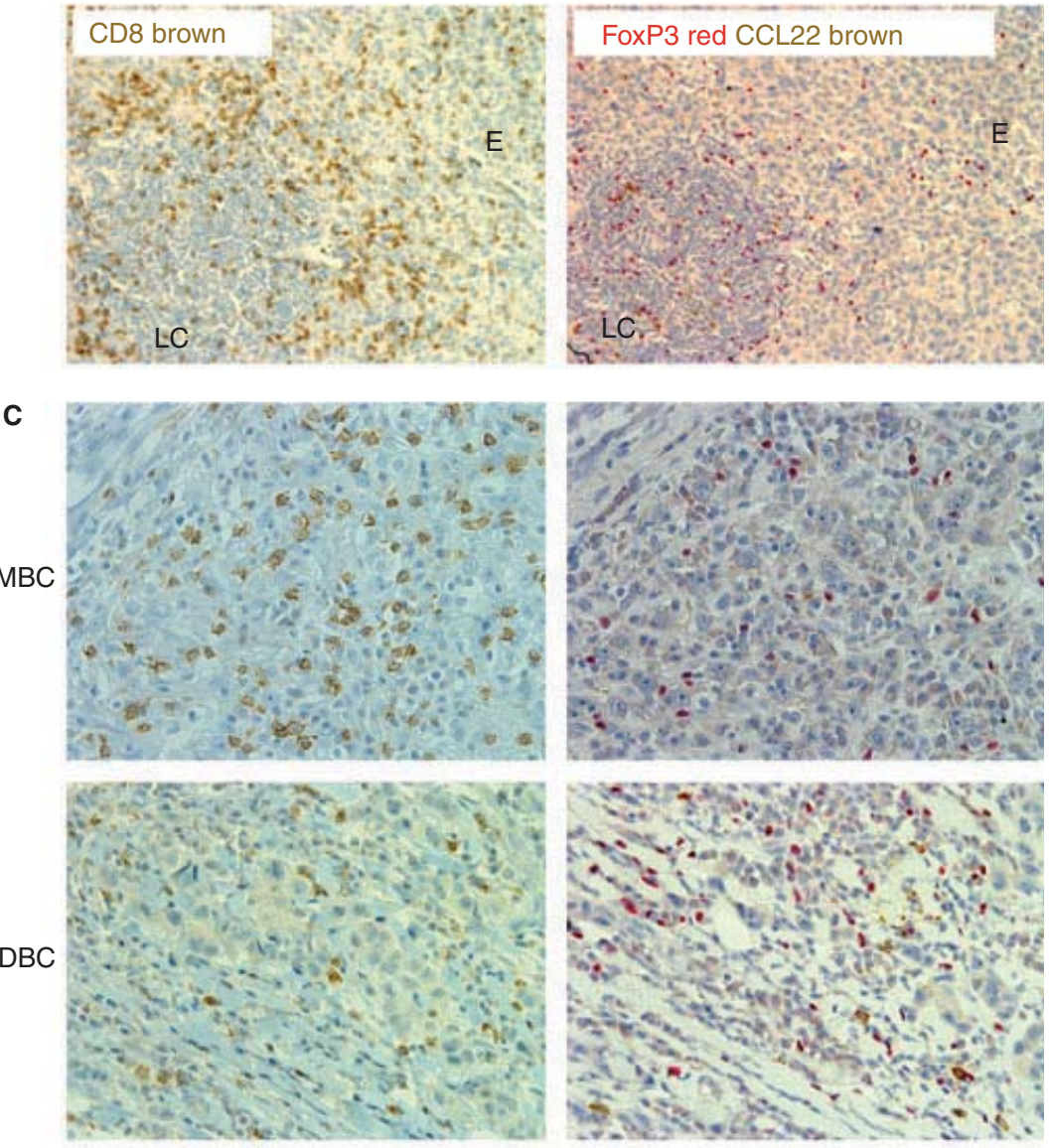
detected by histology, ${ }^{10,17-19}$ flow cytometry, ${ }^{20}$ or polymerase chain reaction (PCR). ${ }^{21,22}$ Two studies on DBC, using tissue microarrays, have shown that the number of tumour-infiltrating FoxP3+ cells is significantly correlated with reduced survival. ${ }^{11,23}$ One recent study on invasive breast cancer, however, did not find a correlation between high Treg numbers and survival, on the basis of multivariate analysis. ${ }^{24}$ The findings in DBC are in line with studies on other types of cancer, where the number of tumour-infiltrating FoxP3+ cells is associated with poor prognosis. ${ }^{25,26}$ The strong infiltration of MBC by FoxP $3+$ cells and the good prognosis of this tumour thus contrast with the observations in other types of human cancer.

In addition to tumour-infiltrating FoxP3+ cells in $\mathrm{MBC}$, we detected high levels of intratumoral CD8+ cells. This is in agreement with previous reports that have described tumour-infiltrating $\mathrm{CD} 3+$ and $\mathrm{CD} 8+$ cells in MBC. 6,15 In contrast to Tregs, tumour infiltration by $\mathrm{T}$ lymphocytes in general is thought to positively influence the survival of cancer patients. In a study on patients with ovarian cancer, the 5-year survival rate was $38 \%$ among patients whose tumours contained CD3+ cells versus $4.5 \%$ for patients without intratumoral $\mathrm{T}$ cells. ${ }^{27}$ Similar findings, based on the examination of intratumoral $\mathrm{T}$ cells, were observed for other types of cancer, such as colorectal carcinoma and melanoma. ${ }^{28,29}$ In the present study, MBCs were abundantly infiltrated by both $\mathrm{CD} 8+$ and FoxP3+ cells, prompting us to determine the ratio of these T-cell subpopulations. Interestingly, we found a clear predominance of CD8+ $\mathrm{T}$ cells, reflected by a mean ratio of $\mathrm{CD} 8+$ to FoxP3+ cells of 2.6 in the tumour epithelium of MBCs. In contrast, equal numbers of $\mathrm{CD} 8+$ and FoxP $3+$ cells were found in the tumour epithelium of DBCs. Although the mean ratio of $\mathrm{CD} 8+$ to FoxP3+ cells showed a highly significant difference between the two types of breast cancer, there was some overlap, as a few individual tumours did note show this pattern. It would be interesting to analyse the clinical outcome of these patients. DBC tissues in our study had relatively low amounts of lymphocytic infiltrate, so it would also be interesting to assess the ratio of $\mathrm{CD} 8+$ to FoxP3+ cells between MBCs and DBCs with prominent inflammation. In summary, the high ratio of intratumoral CD8+ to FoxP3+ cells could contribute to the good prognosis of MBC.

The recruitment of effector $\mathrm{T}$ cells or Tregs into malignant tumours is generally mediated by specific chemokines. CCR4 is a chemokine receptor that is expressed at higher levels on $\mathrm{CD} 4+/$ FoxP $3+$ cells than on other T-cell populations in human blood. ${ }^{12}$ One ligand for CCR4 is CCL22, a chemokine that has been detected in different human tumours. ${ }^{30,31}$ Indeed, tumour homogenates can induce CCL22-dependent migration of Tregs, ${ }^{32,33}$ and the role of CCL22 in the recruitment of Tregs to tumours has also been demonstrated in vivo. ${ }^{10}$ We investigated the expression of this chemokine in different subtypes of breast cancer, and found that all analysed tumours and, in addition, the adjacent healthy breast tissues were infiltrated by CCL22+ myeloid-shaped immune cells. These cells were present in the tumour epithelium, the surrounding connective tissue and, in some cases, in lymphoid clusters, where they were locally associated with FoxP3+ Tregs. Unexpectedly, colocalization of CCL22+ with FoxP3+ immune cells was generally rare, and was limited to lymphoid accumulations. Furthermore, no differences in the number of intratumoral CCL22+ cells were found between MBCs and DBCs, although MBCs contained significantly higher numbers of FoxP3+ Tregs. In addition, no correlation between the numbers of CCL22+ and FoxP3+ cells was seen within the different DBC tissues analysed in this study. This contrasts with a previous report based on reverse-transcriptase PCR analysis that showed a positive correlation between CCL22 and FoxP3 mRNA transcripts in DBC. ${ }^{22}$ Another study on Tregs in DBC, based on tissue microarrays, described a positive correlation between CCL22 and FoxP3 expression in lymphoid infiltrates but not in the tumour parenchyma, which is supported by our observations in DBC. ${ }^{23}$ Thus, in breast cancer, CCL22+ immune cells may attract FoxP3+ Tregs to lymphoid clusters surrounding the tumour bed, but the functional role of CCL22+ immune cells in the epithelium or the stroma of the tumours remains unclear.

Although it has been hypothesized that intratumoral CCL22 may be secreted not only by immune cells but also by the tumour cells themselves, conclusive data demonstrating CCL22 expression by cancer cells in solid tumours have not been available so far. Here, we have clearly shown that, in breast cancer, CCL22+ tumour cells do indeed exist. Whereas almost no CCL22+ tumour cells were found in MBCs, we detected these cells in eight of 34 DBC tissues. Interestingly, in some of these tumours, cancer cells with strong CCL22 expression were found in lymphatic vessels, suggesting that they might have a higher metastatic potential. As CCL22+ cancer cells were not detected in association with FoxP3+ or CD8+ cells, it remains questionable whether these cells are involved in the recruitment of lymphocytes. It will be interesting to find out more about the significance of CCL22 expression by cancer cells. 
In conclusion, our study shows that MBC, despite a good clinical prognosis, is strongly infiltrated by FoxP $3+$ cells. The number of intratumoral CD8+ cells clearly exceeds the number of Tregs in most MBCs, but not in other types of breast cancer. Thus, a predominance of tumour-infiltrating CD8+ over FoxP3+ cells is typically observed in the medullary subtype of breast cancer, and may contribute to its favourable prognosis. This supports the hypothesis that the ratio of intratumoral CD8+ to FoxP3+ cells rather than the absolute number of Tregs is predictive for the clinical outcome of cancer.

\section{Acknowledgements}

We thank Andrea Sendelhofert and Anja Heier for expert technical assistance. This study was supported by grants from the Friedrich Baur Foundation (D. Mayr and D. Anz), an LMUexcellent research professorship (S. Endres), and grants from the German Research Foundation (DFG En 169/7-2 and Graduiertenkolleg 1202) (C. Bourquin and S. Eiber), the excellence cluster CIPS-M 114 (S. Eiber), and BayImmuNet (C. Bourquin and S. Eiber).

\section{Disclosure}

The authors declare no conflicts of interest.

\section{References}

1. Reinfuss M, Stelmach A, Mitus J, Rys J, Duda K. Typical medullary carcinoma of the breast: a clinical and pathological analysis of 52 cases. J. Surg. Oncol. 1995; 60; 89-94.

2. Rapin V, Contesso G, Mouriesse H et al. Medullary breast carcinoma. A reevaluation of 95 cases of breast cancer with inflammatory stroma. Cancer 1988; 61; 2503-2510.

3. Malyuchik SS, Kiyamova RG. Medullary breast carcinoma. Exp. Oncol. 2008; 30; 96-101.

4. Tamiolakis D, Simopoulos C, Cheva A et al. Immunophenotypic profile of tumor infiltrating lymphocytes in medullary carcinoma of the breast. Eur. J. Gynaecol. Oncol. 2002; 23; 433-436.

5. Yakirevich E, Izhak OB, Rennert G, Kovacs ZG, Resnick MB. Cytotoxic phenotype of tumor infiltrating lymphocytes in medullary carcinoma of the breast. Mod. Pathol. 1999; 12; 10501056.

6. Kuroda H, Tamaru J, Sakamoto G, Ohnisi K, Itoyama S. Immunophenotype of lymphocytic infiltration in medullary carcinoma of the breast. Virchows Arch. 2005; 446; 10-14.

7. Vignali DA, Collison LW, Workman CJ. How regulatory T cells work. Nat. Rev. Immunol. 2008; 8; 523-532.

8. Betts GJ, Clarke SL, Richards HE, Godkin AJ, Gallimore AM. Regulating the immune response to tumours. Adv. Drug Deliv. Rev. 2006; 58; 948-961.

9. Kryczek I, Liu R, Wang G et al. FOXP3 defines regulatory T cells in human tumor and autoimmune disease. Cancer Res. 2009; 69; 3995-4000.
10. Curiel TJ, Coukos G, Zou L et al. Specific recruitment of regulatory $\mathrm{T}$ cells in ovarian carcinoma fosters immune privilege and predicts reduced survival. Nat. Med. 2004; 10; 942-949.

11. Bates GJ, Fox SB, Han C et al. Quantification of regulatory T cells enables the identification of high-risk breast cancer patients and those at risk of late relapse. J. Clin. Oncol. 2006; 24; 53735380.

12. Iellem A, Mariani M, Lang R et al. Unique chemotactic response profile and specific expression of chemokine receptors CCR 4 and CCR 8 by CD4(+)CD25(+) regulatory T cells. J. Exp. Med. 2001; 194; 847-853.

13. Sato E, Olson $\mathrm{SH}$, Ahn J et al. Intraepithelial CD8+ tumorinfiltrating lymphocytes and a high $\mathrm{CD} 8+$ / regulatory $\mathrm{T}$ cell ratio are associated with favorable prognosis in ovarian cancer. Proc. Natl Acad. Sci. USA 2005; 102; 18538-18543.

14. Leffers N, Gooden MJ, de Jong RA et al. Prognostic significance of tumor-infiltrating T-lymphocytes in primary and metastatic lesions of advanced stage ovarian cancer. Cancer Immunol. Immunother. 2009; 58; 449-459.

15. Guo X, Fan Y, Lang R et al. Tumor infiltrating lymphocytes differ in invasive micropapillary carcinoma and medullary carcinoma of breast. Mod. Pathol. 2008; 21; 1101-1107.

16. Rakha EA, Aleskandarany M, El-Sayed ME et al. The prognostic significance of inflammation and medullary histological type in invasive carcinoma of the breast. Eur. J. Cancer 2009; 45; 17801787.

17. Ghebeh H, Barhoush E, Tulbah A, Elkum N, Al-Tweigeri T, Dermime S. FOXP3+ Tregs and B7-H1+/PD-1+ T lymphocytes co-infiltrate the tumor tissues of high-risk breast cancer patients: implication for immunotherapy. BMC Cancer 2008; 8; 57.

18. Bohling SD, Allison KH. Immunosuppressive regulatory T cells are associated with aggressive breast cancer phenotypes: a potential therapeutic target. Mod. Pathol. 2008; 21; 1527-1532.

19. Aruga T, Suzuki E, Saji S et al. A low number of tumorinfiltrating FOXP3-positive cells during primary systemic chemotherapy correlates with favorable anti-tumor response in patients with breast cancer. Oncol. Rep. 2009; 22; 273-278.

20. Leong PP, Mohammad R, Ibrahim $\mathrm{N}$ et al. Phenotyping of lymphocytes expressing regulatory and effector markers in infiltrating ductal carcinoma of the breast. Immunol. Lett. 2006; 102; 229-236.

21. Gupta S, Joshi K, Wig JD, Arora SK. Intratumoral FOXP3 expression in infiltrating breast carcinoma: its association with clinicopathologic parameters and angiogenesis. Acta Oncol. 2007; 46; 792-797.

22. Ohara M, Yamaguchi Y, Matsuura K, Murakami S, Arihiro K, Okada M. Possible involvement of regulatory $\mathrm{T}$ cells in tumor onset and progression in primary breast cancer. Cancer Immunol. Immunother. 2009; 58; 441-447.

23. Gobert M, Treilleux I, Bendriss-Vermare N et al. Regulatory T cells recruited through CCL22/CCR 4 are selectively activated in lymphoid infiltrates surrounding primary breast tumors and lead to an adverse clinical outcome. Cancer Res. 2009; 69; 2000-2009.

24. Mahmoud SM, Paish EC, Powe DG et al. An evaluation of the clinical significance of $\mathrm{FOXP} 3(+)$ infiltrating cells in human breast cancer. Breast Cancer Res. Treat. 2010; 127; 99-108.

25. Perrone G, Ruffini PA, Catalano V et al. Intratumoural FOXP3positive regulatory $\mathrm{T}$ cells are associated with adverse prognosis in radically resected gastric cancer. Eur. J. Cancer 2008; 44; 1875-1882.

26. Schreck S, Friebel D, Buettner M et al. Prognostic impact of tumour-infiltrating $\mathrm{Th} 2$ and regulatory $\mathrm{T}$ cells in classical Hodgkin lymphoma. Hematol. Oncol. 2009; 27; 31-39. 
27. Zhang L, Conejo-Garcia JR, Katsaros D et al. Intratumoral T cells, recurrence, and survival in epithelial ovarian cancer. N. Engl. J. Med. 2003; 348; 203-213.

28. Galon J, Costes A, Sanchez-Cabo F et al. Type, density, and location of immune cells within human colorectal tumors predict clinical outcome. Science 2006; 313; 19601964.

29. Clemente CG, Mihm MC Jr, Bufalino R, Zurrida S, Collini P, Cascinelli N. Prognostic value of tumor infiltrating lymphocytes in the vertical growth phase of primary cutaneous melanoma. Cancer 1996; 77; 1303-1310.

30. Mizukami Y, Kono K, Kawaguchi Y et al. CCL17 and CCL22 chemokines within tumor microenvironment are related to accumulation of Foxp $3(+)$ regulatory T cells in gastric cancer. Int. J. Cancer 2008; 122; 2286-2293.

31. Ishida T, Ishii T, Inagaki A et al. Specific recruitment of CC chemokine receptor 4-positive regulatory $\mathrm{T}$ cells in Hodgkin lymphoma fosters immune privilege. Cancer Res. 2006; 66; $5716-5722$.

32. Qin XJ, Shi HZ, Deng JM, Liang QL, Jiang J, Ye ZJ. CCL22 recruits CD4-positive CD25-positive regulatory $\mathrm{T}$ cells into malignant pleural effusion. Clin. Cancer Res. 2009; 15; 2231-2237.

33. Yang ZZ, Novak AJ, Stenson MJ, Witzig TE, Ansell SM. Intratumoral $\mathrm{CD} 4+\mathrm{CD} 25+$ regulatory T-cell-mediated suppression of infiltrating CD4+ T cells in B-cell non-Hodgkin lymphoma. Blood 2006; 107; 3639-3646. 